\title{
Two-color free-electron laser with two orthogonal undulators
}

\author{
G. Dattoli, ${ }^{1}$ N. S. Mirian, ${ }^{2,3}$ E. DiPalma, ${ }^{1}$ and V. Petrillo ${ }^{3}$ \\ ${ }^{1}$ ENEA Centro Ricerche Frascati, via E. Fermi, 45, IT 00044 Frascati, Rome, Italy \\ ${ }^{2}$ School of Particle and Accelerator Physics, Institute for Research in Fundamental Sciences (IPM), \\ 19395-5531 Tehran, Iran \\ ${ }^{3}$ Università degli Studi di Milano, via Celoria 16, IT 20133 Milano, Italy \\ and INFN-Mi, via Celoria 16, IT 20133 Milano, Italy
}

(Received 17 January 2014; published 19 May 2014)

\begin{abstract}
We discuss a two-color self-amplified spontaneous emission free-electron laser (FEL) amplifier where the emission is obtained from two orthogonally polarized undulators with different periods and field intensities. Nonaveraged and averaged equations are compared. The two radiations have not only different frequencies, but also different polarizations, while the total length of the device does not change with respect to usual single-color FELs. The wavelengths of two different colors can be changed by choosing different periods, while variation in the magnetic strengths can be used to modify the gain lengths.
\end{abstract}

DOI: 10.1103/PhysRevSTAB.17.050702

PACS numbers: 41.60.Cr

\section{INTRODUCTION}

The development of radiation sources characterized by high flux and tunability opens the way for a real breakthrough in many scientific and technical fields. One of the most promising formats in which radiation can be delivered to users is in the form of packets containing two different spectral lines with adjustable time separation. In particular, by means of two-color $\mathrm{x}$ rays it is possible to perform pump and probe experiments of structural dynamics [1,2], thus probing matter on the atomic scale in space and time [3,4]. Moreover, in x-ray technology, the color components, containing extra information, allows one to discriminate the chemical composition of the absorbing tissues [5,6], with significant developments in the field of screening or diagnostic clinical imaging. Free-electron lasers (FELs) [7-10] are considered among the most performing radiation sources. Experiments on dual frequency production have been recently carried out on them with different methods [11-18] in various regimes of radiation frequency. Some of the initially proposed designs [19-21] were based on the use of staggered undulator magnets with different values of deflecting parameters to achieve lasing at two distinct wavelengths [22-27], an idea recently revisited and demonstrated in the $\mathrm{x}$ rays' range at the FEL Linac Coherent Light Source (LCLS) [8,11]. Another option, recently demonstrated at the FERMI soft $\mathrm{x}$-ray seeded FEL [10], involves the use of either a chirped or a two-color seed laser $[13,14]$. A different approach is to inject in the undulator a multienergy electron beam [28] resonating at two different

Published by the American Physical Society under the terms of the Creative Commons Attribution 3.0 License. Further distribution of this work must maintain attribution to the author(s) and the published article's title, journal citation, and DOI. wavelengths, allowing the control of frequency and time separation ranges of the FEL pulse, while maintaining similar saturated power levels and minimal undulator length $[12,15,29]$, as done at SPARC (Sorgente Pulsata e Amplificata di Radiazione Coerente). In this paper, we analyze the operation with a further different scheme: the FEL emission is obtained from two orthogonally polarized undulators with different periods and field intensities. In this case, the two colors have also different polarizations, while the total length of the device does not change much with respect to usual single-color FELs. The possibility of having orthogonal polarizations with comparable intensities opens various possibilities to get insights into internal organization and orientation in space of molecules, taking advantage of the selective excitation of the molecular fluorescence by differently polarized beams, significantly improving the techniques based on fluorescence anisotropy [30] and dichroism [31]. Such a radiation beam can be realized, whenever possible, by means of the use of polarizers, or by projecting an ad hoc undulator, depending on the different experimental situations and on the needed wavelengths. An undulator with crossed polarizations in a deltalike magnetic structure [32] (see Fig. 1) has been already constructed and measured [33], and the extension to the configuration discussed in this paper is in progress.

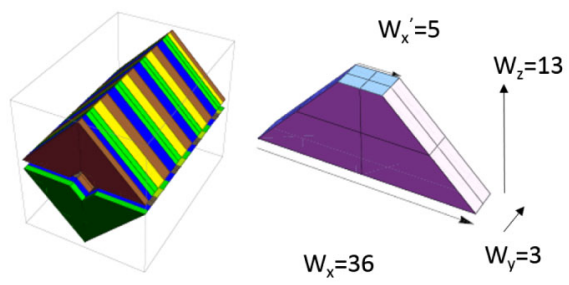

FIG. 1. Schematic picture of the deltalike magnetic undulator. 


\section{EQUATION MODEL}

The FEL undulator field can be modeled as two linear undulators orthogonally polarized with periods $\lambda_{01,02}=2 \pi / k_{01,02}$ and deflecting parameters $K_{1,2}=$ $\left|e B_{1,2} \lambda_{01,02} / m c^{2}\right|$, described by the expression $\underline{B}_{w}=$ $-B_{2} \sin \mathrm{k}_{02} z \underline{e}_{x}+B_{1} \sin \mathrm{k}_{01} z \underline{e}_{y}$. In the radiation potential $\underline{A}=-i\left[A_{1} e^{i\left(k_{1} z-\omega_{1} t\right)} \underline{e}_{x}+A_{2} e^{i\left(k_{2} z-\omega_{2} t\right)} \underline{e}_{y}\right], \quad A_{1,2}$ are slow complex amplitudes, $k_{1,2}=2 \pi / \lambda_{1,2}$ and the two wavelengths satisfy the relevant resonance conditions $\lambda_{1,2}=\frac{\lambda_{01,02}}{2 \gamma_{0}^{2}}\left(1+K_{1}^{2} / 2+K_{2}^{2} / 2\right)$. In the following, we will assume $n \lambda_{01}=m \lambda_{02}$, which permits us to treat the cases of a harmonic relation between $\lambda_{01}$ and $\lambda_{02}$ and of rational $m / n$.

The momentum equations for the $j$ th electron are

$$
\begin{aligned}
\frac{d\left[p_{x, y}\right]_{j}}{d t}= & e \beta_{z j} B_{1,2} \sin \mathrm{k}_{01,02} z_{j} \\
& -e k_{1,2}\left(1-\beta_{z j}\right) A_{1,2} e^{i\left[\alpha_{1,2}\right]_{j}}+c c, \\
\frac{d p_{z j}}{d t}= & -e \beta_{y j} B_{2} \sin \mathrm{k}_{02} z_{j}-e k_{2} \beta_{y j} A_{2} e^{i \alpha_{2 j}}+c c \\
& -e \beta_{x j} B_{1} \sin \mathrm{k}_{01} z_{j}-e k_{1} \beta_{x j} A_{1} e^{i \alpha_{1 j}}+c c,
\end{aligned}
$$

with $\left[\alpha_{1,2}\right]_{j}=k_{1,2} z_{j}-\omega_{1,2} t$.

The essential characteristics of the systems can be obtained by means of a one-dimensional treatment.

From the potential equation, assigning the transverse currents in terms of the particle density $\bar{n}$ as $J_{x, y}=$ $-\sum_{j} e c\left[\beta_{x, y}\right]_{j} \bar{n} \delta\left(z-z_{j}\right)$ and using the slowly varying envelope approximation, in the one-dimensional model, two differential equations

$$
\frac{\partial}{\partial z} A_{1,2}+\frac{1}{c} \frac{\partial}{\partial t} A_{1,2}=\frac{2 \pi e \bar{n}}{k_{1,2}} \sum_{j}\left[\beta_{x, y}\right]_{j} \delta\left(z-z_{j}\right) e^{-i\left[\alpha_{1,2}\right]_{j}}
$$

with $\left[\beta_{x, y}\right]_{j}=\left[v_{x, y}\right]_{j} / c$ are obtained. Differently from the case of two FEL waves with distinct wavelengths, but same polarization, the equations for the potential result coupled only through the electron longitudinal dynamics appearing in $\alpha_{1,2}$, while the transverse motions remain decoupled. Following the Colson's analysis [34], the zeroorder dimensionless transverse velocities are $\left[\beta_{x, y}\right]_{j}=$ $-\frac{K_{1,2}}{\gamma_{0}} \cos \left(k_{01,02} z_{j}\right) \approx-\frac{K_{1,2}}{\gamma_{0}} \cos \left(\omega_{01,02} t\right)$, and the treedimensional trajectories of the electrons inside the undulator are given by

$$
\begin{aligned}
\underline{r}_{j}= & \beta_{0 j} c t \underline{e}_{z}-\frac{K_{1}}{k_{01} \gamma_{0 j}} \sin \left(\omega_{01} t\right) \underline{e}_{x}-\frac{K_{2}}{k_{02} \gamma_{0 j}} \sin \left(\omega_{02} t\right) \underline{e}_{y} \\
& -\frac{\lambda_{01}}{16 \pi} \frac{K_{1}^{2}}{\gamma_{0 j}^{2}} \sin \left(2 \omega_{01} t\right) \underline{e}_{z}-\frac{\lambda_{02}}{16 \pi} \frac{K_{2}^{2}}{\gamma_{0 j}^{2}} \sin \left(2 \omega_{02} t\right) \underline{e}_{z},
\end{aligned}
$$

giving, at zero order, $\quad z \approx \beta_{0 j} c t-\frac{\xi_{1}}{k_{1}} \sin \left(2 \omega_{01} t\right)-$ $\frac{\xi_{2}}{k_{2}} \sin \left(2 \omega_{02} t\right)$, with $\xi_{1,2}=\frac{K_{1,2}^{2}}{4\left(1+K_{1}^{2} / 2+K_{2}^{2} / 2\right)}$. By defining the phases $\left[\theta_{1,2}\right]_{j}=\omega_{01,02} t+k_{1,2} \beta_{z j} c t-\omega_{1,2} t$ and introducing a double average in the $x$ and $y$ directions over a module $L$ multiple of the undulator periods $L=n \lambda_{01}=m \lambda_{02}$, the equation for the energy becomes

$$
\frac{d \gamma_{j}}{d t}=\frac{e}{4 m c}\left(\frac{K_{1} k_{1}}{\gamma_{0 j}} \mathcal{F}_{1} e^{i \theta_{1 j}} A_{1}+\frac{K_{2} k_{2}}{\gamma_{0 j}} \mathcal{F}_{2} e^{i \theta_{2 j}} A_{2}+c c\right),
$$

where, using the integral expression of the Bessel's functions $J_{n}(x)=\frac{1}{2 \pi} \int_{-\pi}^{\pi} d \tau e^{i(x \sin \tau-n \tau)}$, we inserted the Bessel coupling factors modified for the case of two undulators $\mathcal{F}_{1,2}=J_{0}\left(\frac{k_{1,2} \xi_{2,1}}{k_{2,1}}\right)\left[J_{0}\left(\xi_{1,2}\right)-J_{1}\left(\xi_{1,2}\right)\right]$. Operating the same average on the potential equations leads to

$$
\frac{\partial A_{1,2}}{\partial z}+\frac{1}{c} \frac{\partial A_{1,2}}{\partial t}=\frac{e \bar{n} \pi K_{1,2}}{k_{1,2} \gamma_{0}} \mathcal{F}_{1,2} \sum e^{-i\left[\theta_{1,2}\right]_{j}} .
$$

In the limit of only one undulator, $K_{2}=0\left(J_{0}(0)=1\right)$, the usual Colson's equations are retrieved.

In the universal scaling notation [35], the normalized fields $a_{1,2}=\frac{\omega_{1}}{\omega_{p} \sqrt{\rho_{1} \gamma_{0}}} \frac{e A_{1,2}}{m c^{2}}$ and $\Gamma_{i}=\frac{\gamma_{i}-\gamma_{0 i}}{\rho_{1} \gamma_{0 i}}$ satisfy the scaled equations

$$
\begin{gathered}
\frac{d \Gamma_{i}}{d \tau}=e^{i \theta_{1 i}} a_{1}+\frac{\mathcal{F}_{2}}{\mathcal{F}_{1}} \frac{K_{2}}{K_{1}} \frac{k_{02}}{k_{01}} e^{i \theta_{2 i}} a_{2}+c c, \\
\frac{\partial a_{1}}{\partial \zeta}+\frac{1}{c} \frac{\partial a_{1}}{\partial \tau}=\sum e^{-i \theta_{1 i}}, \\
\frac{\partial a_{2}}{\partial \zeta}+\frac{1}{c} \frac{\partial a_{2}}{\partial \tau}=\frac{K_{2} k_{01}}{K_{1} k_{02}} \frac{\mathcal{F}_{2}}{\mathcal{F}_{1}} \sum e^{-i \theta_{2 i}},
\end{gathered}
$$

while the phases evolve according to

$$
\frac{d \theta_{1 i}}{d \tau}=\Gamma_{i}, \quad \frac{d \theta_{2 i}}{d \tau}=\frac{k_{02}}{k_{01}} \Gamma_{i},
$$

$\rho_{1}=\frac{1}{\gamma_{0}}\left[\frac{\omega_{p} K_{1} \mathcal{F}_{1}}{8 \omega_{01}}\right]^{2 / 3}$ being the FEL parameter and $\omega_{p}$ the plasma frequency.

The gain lengths of the two polarizations, when $m \neq n$, are therefore

$$
L_{g 1}=\frac{\lambda_{01}}{4 \pi \sqrt{3} \rho_{1}}=\left(\frac{\lambda_{01}}{\lambda_{02}}\right)^{1 / 3}\left(\frac{\mathcal{F}_{2} K_{2}}{\mathcal{F}_{1} K_{1}}\right)^{2 / 3} L_{g 2}
$$

\section{ANALYTICAL AND NUMERICAL DATA}

Equations (1) and (2), obtained in the nonaveraged treatment, and Eqs. (6)-(9), averaged on the undulator period, have been solved numerically with two independent codes, in the time-independent approximation, showing an overall satisfactory agreement between the two models, as always in similar situations [36,37]. In particular, the code 

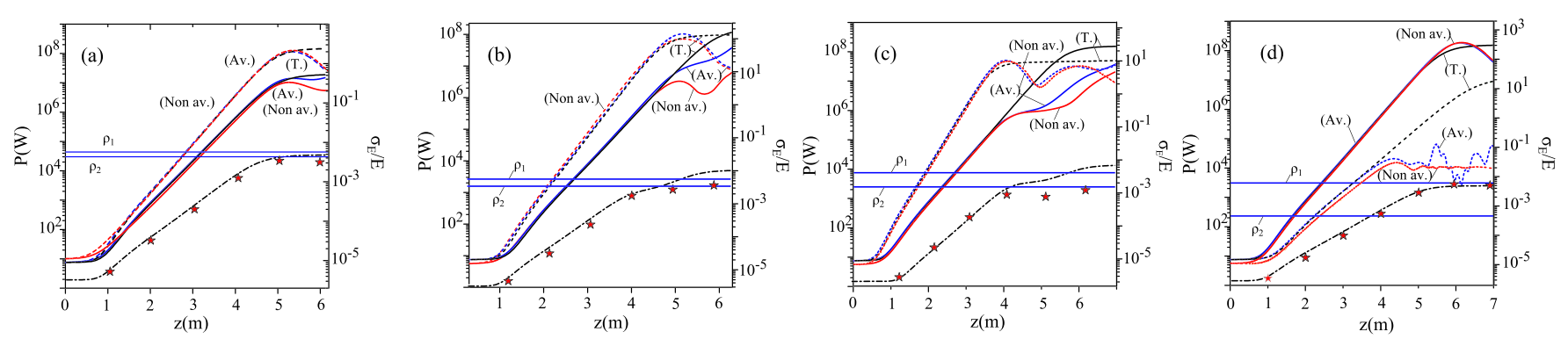

FIG. 2. Left axis: power $P(W)$ in the $x$ (solid curves) and $y$ (dashed curves) polarizations vs $z(m)$. Comparison between nonaveraged (red curves) and averaged (blue curved) model for (a) $m / n=1.5$, (b) $m / n=2$, (c) $m / n=5$, and (d) $m / n=10$. In black, the logistic map $P_{1,2}^{T}$. On right axis, induced energy spread $\frac{\sigma_{E}}{E}$ from phase space (red stars) and theoretical (black curves). $\lambda_{01}=2.8 \mathrm{~cm}$, $K_{2}=K_{1}=2.1$, the electron current $I=100 \mathrm{~A}$, and $\gamma=300$.

for the solution of the nonaveraged equations follows the line of the code MEDUSA [36,37] and is based on secondorder Runge-Kutta method and Gauss quadrature integration for the calculation of the bunching factor. The code for the solution of the averaged equations is based on the fourth-order Runge-Kutta method. Both codes use the quiet start. The parameters of the simulations are similar to the SPARC's $[38,39]$, with a value of $\rho_{1}=5.47 \times 10^{-3}$ and $n \lambda_{01}=m \lambda_{02}$.

The comparison between the solutions of nonaveraged (red line) and averaged (blue line) equations is shown in Fig. 2 for (a) $m / n=1.5$, (b) $m / n=2$, (c) $m / n=5$, and (d) $m / n=10$.

The agreement is indeed significant during the growth, up to the onset of saturation, where, instead, discrepancies arise when the two waves have similar intensity [case (a)], probably due to the differences in the sets of equations and to the different integration methods. Black curves, labeled with $(\mathrm{T})$, represent the logistic map proposed in [40] $P_{1,2}^{T}=P_{01,02} A_{1,2} e^{\left(0.223 t / Z_{1,2}\right)} /\left[1+\frac{P_{01,02}}{P_{s 1,2}}\left(A_{1,2}-1\right)\right]$ with $P_{s 1,2}=1.42 \rho_{1,2} P_{b}, A_{1,2}=\left[\frac{1}{3}+\frac{2}{9} \cosh \left(\frac{t}{L_{g 1,2}}\right)+\frac{4}{9} \cos \left(\frac{\sqrt{3} t}{2 L_{g 1,2}}\right) \times\right.$ $\left.\cosh \left(\frac{t}{2 L_{g 1,2}}\right)\right], Z_{1,2}=1.066 L_{g} \log \left(9 \frac{P_{s 1,2}}{P_{01,2}}\right), P_{b}$ being the beam power. The previous expressions fit very accurately lethargy, growth, and gain lengths of both waves. The
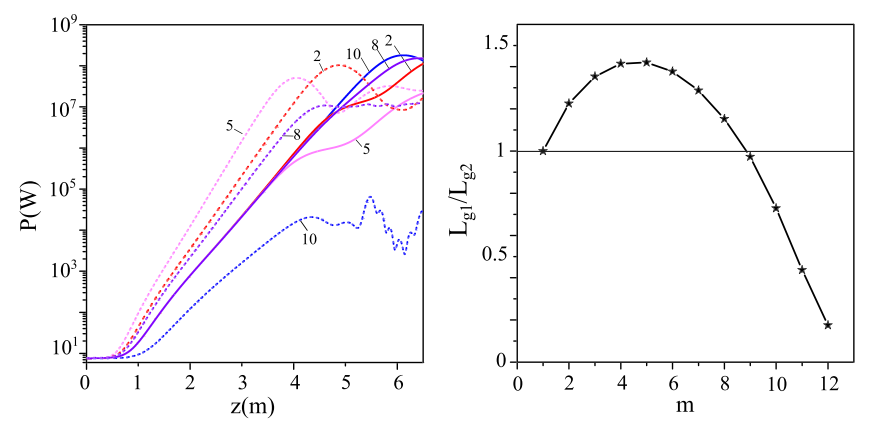

FIG. 3. (a) $P(W)$ as a function of the coordinate $z(m)$ along the undulator for $m / n=2$ (red curve), 5 (light magenta curve), 8 (violet curve), and 10 (blue curve). Solid line denotes $x$ polarization; dashed line denotes $y$ polarization. (b) $L_{g 1} / L_{g 2}$ as a function of $m / n$. saturation value, instead, is of the same order only for one of the two polarizations, due to the interaction between them occurring when the power is large, an effect that is not accounted in $P_{1,2}^{T}$. In fact, an increase in the energy spread $\sigma_{E}$ is induced, as can be seen in Fig. 2, where the relative value $\sigma_{E} / E$, computed by the phase space, is presented together with the quadratic sum of the two terms:

$$
\frac{\sigma_{1,2}}{E}=\frac{3}{2} \sqrt{\frac{\rho_{1,2} P_{01,2}}{P_{b}}} \sqrt{\frac{A_{1,2}}{1+1.24 \frac{P_{01,2}}{P_{s 1,2}}\left(A_{1,2}-1\right)}} .
$$

The energy spread grows up to $1.6 \rho_{1}$, and produces the anticipated saturation of the higher frequency radiation when $\frac{\sigma_{E}}{E} \approx \rho_{2}$. The final value of power $P_{s}$ is reached after a series of oscillations, due to the phenomenon of the mutual bunching as described in Ref. [29].

Figure 3(a) compares the powers for few values of $m / n$ between 2 and 10, obtained with the averaged model. The

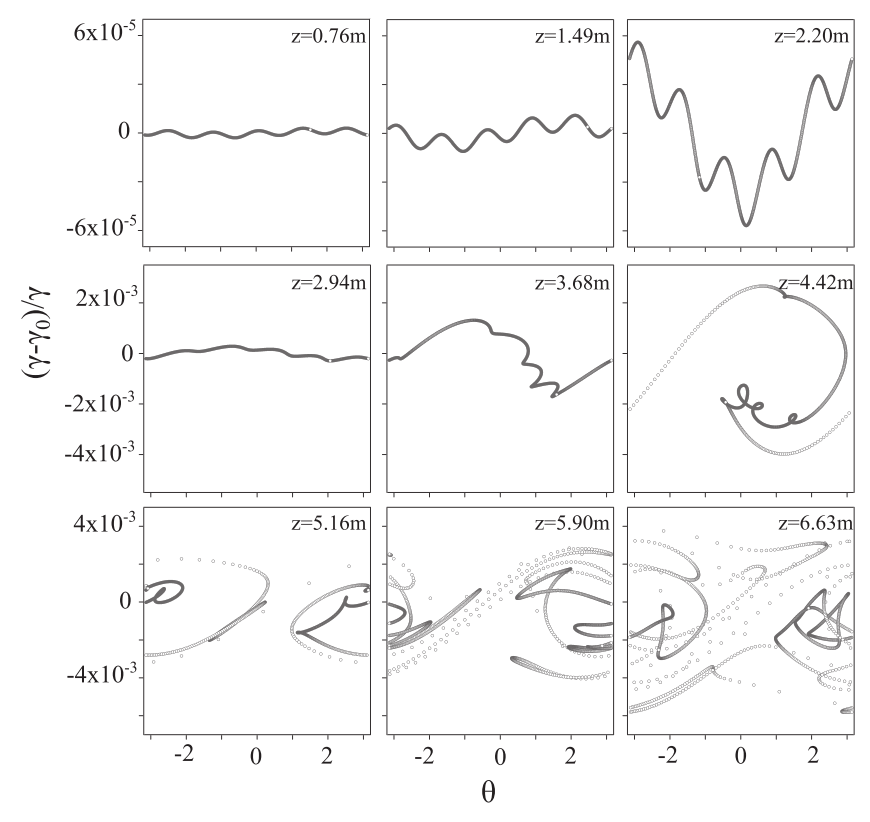

FIG. 4. Electron longitudinal phase space for $m / n=5$ and different values of the coordinate $z$ along the undulator. 

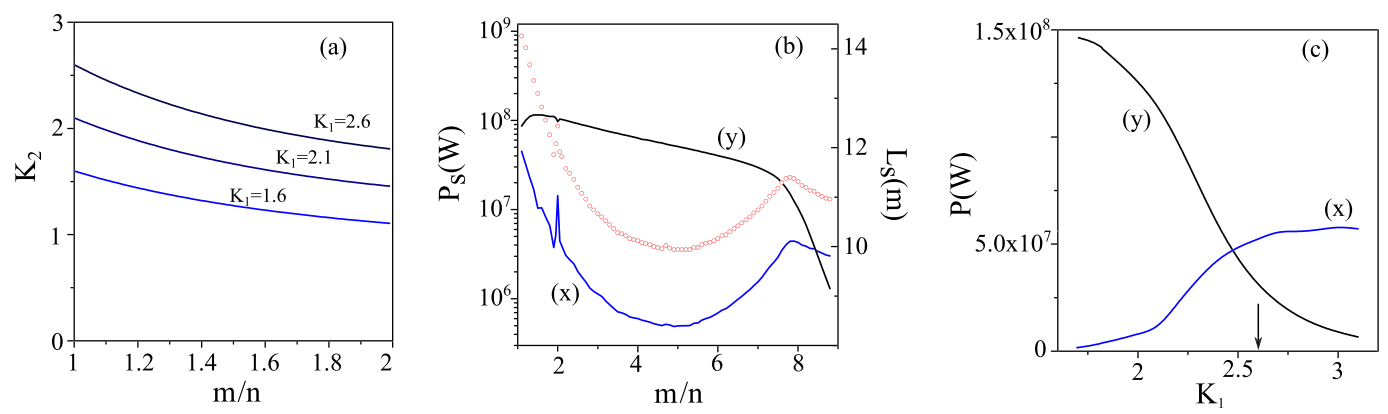

FIG. 5. (a) $K_{2}$ solution of the relation $L_{g 1}=L_{g 2}$ as a function of $m / n$ and for few values of $K_{1}$. (b) Left axis: in black, first peak value of the power $P_{s}$; in blue, power of the $x$ polarization at $L_{s}$ as a function of $m / n$. Right axis: red circle, saturation length $L_{s}$ of the $y$ polarization, reaching first the saturation. (c) In blue, power $P$ of $x$ and, in black, $y$ polarizations at varying values of $K_{1}$, with $K_{2}=2.1$ and $m / n=1.5$. The powers are both evaluated at the first peak of saturation of the $y$ polarization, which is going first at saturation in this regime. The arrow indicates the point where the two gain lengths are the same.

graph of $L_{g 1} / L_{g 2}$, shown in Fig. 3(b), confirms that from $m=1$ to about $m=9$ the largest frequency wave has a shorter gain length, while for $m$ larger than 9 the opposite occurs.

The longitudinal phase space of the electrons is presented in Fig. 4 for $m / n=5$ at different values of the coordinate $z$ along the undulator. After a period of lethargy (first row), the formation of the bunching on both wavelengths (second row) induces the exponential growth of both polarizations. After that, the phase space begins to develop chaotic patterns (third row), corresponding to deep saturation.

The frequencies of the two polarizations can be approached by varying the factor $m / n$. In the range around $m / n \approx 1$, the system produces two waves of close frequencies, with the largest one characterized by a shorter gain length. Saturation lengths and powers are very similar and can be made closer by choosing the values of $K_{1}$ and $K_{2}$ in such a way to rend the gain lengths equal. The solution of the equation $L_{g 1}=L_{g 2}$, i.e., $\left(\frac{\lambda_{01}}{\lambda_{02}}\right)\left(\frac{\mathcal{F}_{2} K_{2}}{\mathcal{F}_{1} K_{1}}\right)^{2}=1$, is given in Fig. 5(a) for $m / n$ between 1 and 2 and for few values of $K_{1}$.

Figure 5(b) presents, as a function of $m=n$, the first peak value of the power $P_{s}$ and the saturation length $L_{s}$ of the $y$ polarization, that, in the range considered, is the first wave reaching saturation. The power of the other polarization, evaluated at $L_{s}$, is also shown. Since after the first peak other maxima occur, both waves, after this length, can reach larger values of power. Around the second harmonics, the strong harmonic bunching causes a larger interaction between the two waves with an increase of the $x$ polarization power.

For balancing the level of power along the two directions at the end of the FEL, the magnetic strength of one of the two undulators can be varied. The effect is an overall decreasing of the total power but with a more balanced distribution between the two polarizations, as shown in Fig. 5(c), where $K_{1}$ has been varied at fixed value of $K_{2}$. The arrow indicates the point where the two gain lengths are equal. Around this point, the growth of the two polarizations are synchronous, but, because the saturation lengths are different, the power level reached when the first of the two waves is saturating remains different.

If one of the polarizations is not seeded, the behavior is different if the factor $m / n$ is integer or not.

This is presented in Fig. 6, where the cases $m / n=1.5$ [box (a)], $m / n=2$ [box (b)], and $m / n=3$ [box(c)] have been analyzed by comparing a case (i) where both waves are starting from seed and another case (ii) where the $y$ polarization starts from zero. When $m / n$ is integer [cases (b) and (c)] the growth of the $y$ polarization (dashed curves), when not seeded (ii), begins from zero and increases with the relevant gain length as in the other case (i). In a further stage of the evolution, when the power is large enough, the growth rate increases according to the
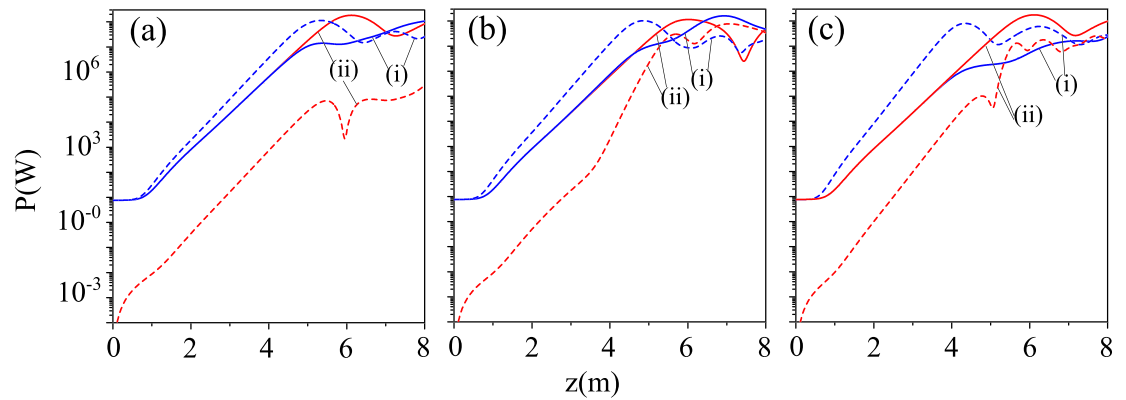

FIG. 6. $P(W)$ as a function of $z(m)$ for (a) $m / n=1.5$, (b) $m / n=2$, and (c) $m / n=3$. (i) Blue curves: $x$ and $y$ polarizations both starting from seed. (ii) Red curves: $y$ polarization starting from zero. Solid lines: $x$ polarization; dashed lines: $y$ polarization. 
phenomenon of the nonlinear harmonic generation that results from the strong electron beam microbunching present at the fundamental wavelength [23,41]. In this case, the harmonics experience a growth similar to that of the fundamental with gain lengths scaling as the inverse of the harmonic number $(1 / \mathrm{m})$. In the case of noninteger $m / n$, this effect does not occur, due to the initial bunching related to the electron distribution.

\section{CONCLUSIONS}

The case of two orthogonal undulators with different polarizations and periods have been studied. Nonaveraged and averaged equations have been written and integrated. These two models present a significant agreement with regard to lethargy, growth, and gain length of the radiation, while discrepancies appear in saturation. A theoretical logistic map fits well the simulations. The use of this kind of device permits one to produce two-color radiation with an easy control of the frequencies and with different polarizations, while the total length of the device does not change with respect to usual single-color FELs. The possibility of changing independently the strength of the two magnetic fields allows one to rule the final power and the saturation length.

[1] F. Tavella, N. Stojanovic, G. Geloni, and M. Gensch, Nat. Photonics 5, 162 (2011).

[2] Y. Ding, F. J. Decker, P. Emma, C. Feng, C. Field, J. Frisch, Z. Huang, J. Krzywinski, H. Loos, J. Welch, J. Wu, and F. Zhou, Phys. Rev. Lett. 109, 254802 (2012).

[3] C. M. Guenther, B. Pfau, R. Mitzner, B. Siemer, S. Roling, H. Zacharias, O. Kutz, I. Rudolph, D. Schondelmaier, R. Treusch, and S. Eisebitt, Nat. Photonics 5, 99 (2011).

[4] A. H. Zewail, J. Phys. Chem. A 104, 5660 (2000).

[5] I. Willekens, B. Dierickx, N. Buls, C. Breucq, A. Schiettecatte, J. de Mey, C. Bourgain, Eur. Conf. Radiol., C-2119 (2011).

[6] I. Willekens, B. Dierickx, N. Buls, C. Breucq, A. Schiettecatte, J. de Mey, and C. Bourgain, European Society for Radiography, Vienna, 2011.

[7] W. Ackermann et al., Nat. Photonics 1, 336 (2007).

[8] P. Emma et al., Nat. Photonics 4, 641 (2010).

[9] H. Tanaka et al., Nat. Photonics 6, 540 (2012).

[10] E. Allaria et al., Nat. Photonics 6, 699 (2012).

[11] A. A. Lutman, R. Coffee, Y. Ding, Z. Huang, J. Krzywinski, T. Maxwell, M. Messerschmidt, and H.-D. Nuhn, Phys. Rev. Lett. 110, 134801 (2013).

[12] V. Petrillo, M. P. Anania, M. Artioli, A. Bacci, M. Bellaveglia, E. Chiadroni et al., Phys. Rev. Lett. 111, 114802 (2013).

[13] E. Allaria, F. Bencivenga, R. Borghes, F. Capotondi et al., Nat. Commun. 4, 2476 (2013).

[14] B. Mahieu, E. Allaria, D. Castronovo, M. B. Danailov et al. Opt. Express 21, 22728 (2013).

[15] A. Marinelli, A. A. Lutman, J. Wu, D. Ratner, S. Gilevich, F. J. Decker, J. Turner, H. Loos, Y. Ding, J. Krzywinski, Y. Feng, H. D. Nuhn, J. Welch, T. Maxwell, C. Behrens,
R. Coffee, Z. Huang, and C. Pellegrini, in Proceedings of FEL Conference, New York, USA, 2013, edited by C. Scholl (BNL) and V.R.W. Schaa (GSI) (2013), WEPSO40.

[16] A. Marinelli, A. A. Lutman, J. Wu, Y. Ding, J. Krzywinski, H.-D. Nuhn, Y. Feng, R. N. Coffee, and C. Pellegrini Phys. Rev. Lett. 111, 134801 (2013).

[17] C. Ronsivalle et al., New J. Phys. 16, 033018 (2014).

[18] E. Chiadroni et al. (to be published).

[19] H. A. Schwettman and T. I. Smith, J. Opt. Soc. Am. B 6, 973 (1989).

[20] D. Iracane and P. Bamas, Phys. Rev. Lett. 67, 3086 (1991).

[21] F. Ciocci, G. Dattoli, L. Giannessi, A. Torre, and G. Voykov, Phys. Rev. E 47, 2061 (1993).

[22] D. A. Jaroszynski, R. Prazeres, F. Glotin, and J. M. Ortega, Phys. Rev. Lett. 72, 2387 (1994).

[23] S. G. Biedron, R. Bartolini, F. Ciocci, G. Dattoli, W. M. Fawley, G. Felici, H. P. Freund, H-D. Nuhn, P. L. Ottaviani, and A. Renieri, SPIE paper No. 4632-35, 2002.

[24] G. Dattoli, L. Giannessi, P. L. Ottaviani, H. P. Freund, S. G. Biedron, and S. Milton, Nucl. Instrum. Methods Phys. Res., Sect. A 495, 48 (2002).

[25] G. Dattoli, V. V. Mikhailin, P. L. Ottaviani, and K. V. Zhukovsky, J. Appl. Phys. 100, 084507 (2006).

[26] S. G. Biedron, G. Dattoli, W. M. Fawley, H. P. Freund, Z. Huang, K. J. Kim, S. V. Milton, H.-D. Nuhn, P. L. Ottaviani, and A. Renieri, Phys. Rev. STAccel. Beams 5, 030701 (2002).

[27] H. Jeevakhan and G. Mishra, Nucl. Instrum. Methods Phys. Res., Sect. A 656, 101 (2011).

[28] M. Ferrario et al., Nucl. Instrum. Methods Phys. Res., Sect. A 637, S43 (2011).

[29] F. Ciocci, G. Dattoli, S. Pagnutti, A. Petralia, E. Sabia, P. L. Ottaviani, M. Ferrario, F. Villa, and V. Petrillo, Phys. Rev. Lett. 111, 264801 (2013).

[30] J. R. Lakowicz, Principles of Fluorescence Spectroscopy (Springer, New York, 2006), 3rd ed., Chap. 10-12.

[31] A. K. Kazansky, A. V. Grigorieva, and N. M. Kabachnik Phys. Rev. A 85, 053409 (2012).

[32] A. B. Temnykh, Phys. Rev. ST Accel. Beams 11, 120702 (2008).

[33] E. Sabia, F. Ciocci, G. Dattoli, M. Del Franco, E. DiPalma, L. Giannessi, M. Quattromini, I. P. Spassovsky, V. Surrenti, G. Soregaroli, M. Tedeschi, R. Geometrante, M. Zambelli, M. Kokole, and T. Milharčič, in Proceedings of FEL Conference, New York, USA, 2013, edited by C. Scholl (BNL) and V. R. W. Schaa (GSI) (2013).

[34] W. B. Colson, IEEE J. Quantum Electron. 17, 1417 (1981).

[35] R. Bonifacio, C. Pellegrini and L. Narducci, Opt. Commun. 50, 373 (1984).

[36] H. P. Freund, S. G. Biedron, and S. V. Milton, IEEE J. Quantum Electron. 27, 243 (2000).

[37] H. P. Freund and P. G. O' Shea, Phys. Rev. Lett. 84, 2861 (2000).

[38] M. Ferrario, D. Alesini, M. P. Anania, A. Bacci et al., Nucl. Instrum. Methods Phys. Res., Sect. B 309,183 (2013).

[39] L. Giannessi et al., Phys. Rev. ST Accel. Beams 14, 060712 (2011).

[40] G. Dattoli, P. L. Ottaviani, and S. Pagnutti, Report No. ENEA RT/2007/40/FIM.

[41] L. Giannessi et al. Phys. Rev. Lett. 108, 164801 (2012). 